\title{
AÇÃO DO AGROSTEMIN SOBRE A ALTURA E O NÚMERO DE FOLHAS DE PLANTAS DE SOJA (Glycine max L. MERRILL cv. IAC-8)
}

\author{
A.A.H. FERNANDES; J.D. RODRIGUES; S.D. RODRIGUES \\ Dep. de Botânica do Instituto de Biociências/UNESP - 18618-000-Botucatu,SP.
}

\begin{abstract}
RESUMO: $O$ presente estudo teve por finalidade avaliar o efeito de um estimulante vegetal, o Agrostemin, sobre a altura e o número de folhas das plantas de soja (Glycine max L. MERRILL cv. IAC-8). 0 experimento foi conduzido em casa de vegetação. Foram estudados seis tratamentos, correspondentes ass seguintes dosagens, épocas e formas de aplicação: testemunha (T1); 0,125g Agrostemin/100g sementes (T2); 0,125g Agrostemin/100g semente e pulverização foliar à $333 \mathrm{ppm}(\mathrm{T} 3) ; 0,125 \mathrm{~g}$ Agrostemin $/ 100 \mathrm{~g}$ sementes e pulverização foliar à $500 \mathrm{ppm}$ (T4); pulverização foliar à $333 \mathrm{ppm}$ (T5) e pulverização foliar à $500 \mathrm{ppm}$ (T6). Realizaram-se três coletas de plantas, com intervalo de 14 dias. 0 experimento foi en delineamento inteiramente casualizado, utilizando-se duas análises de variância com desdobramento em efeitos de regressão. Atraves dos resultados obtidos concluiu-se que o Agrostemin quando aplicado via semente (T2) ou via foliar à $500 \mathrm{ppm}$ (T6), aumentou a altura e o número de folhas. $\Lambda 0$ aplicar o produto via semente mais via foliar à $500 \mathrm{ppm}$ (T4), estas medidas diminuiram.
\end{abstract}

Descritores: Glycine max L., Agrostemin, estimulante vegetal.

\section{EFFECTS OF AGROSTEMMIN ON NUMBER OF LEAVES AND IIEIGIIT OF SOYBEAN PLANTS}

\begin{abstract}
ARSTRACT: A greenhouse experiment was conducted to evaluate the effects of Agrostemmin (plant stimulant) on height and number of leaves of soybean plants (Glycine max L Merril cv. I AC-8). Six treatments were studied: check (T1); 0,125g Agrostemmin/100g seed (T2); 0,125g Agrostemmin/100 seed and foliar spraying 333 ppm (T3); 0,125g Agrostemmin/100g seed and foliar spraying $500 \mathrm{ppm}$ (T4); foliar spraying $333 \mathrm{ppm}$ (T5) and foliar spraying $500 \mathrm{ppm}$ (T6). Three samplings were made at 14 day intervals. The experimental layout was completely randomized, using two variance, testing regression effects. Agrostemmin applied to the seed (T2) or sprayed on the leaves at the rate of $500 \mathrm{ppm}$ (T6) increased height and number of leaves of soybean, whereas the combination of $A$ grostemmin applied to the seeds and sprayed at $500 \mathrm{ppm}(\mathrm{T} 4)$ decreased those values.
\end{abstract}

Key Words: Glycine max L., Agrostemmin, plant stimulant.

\section{INTRODUÇÃO}

De acordo com CASTRO (1981), poucos trabalhos abordam aspectos fisiológicos da planta de soja, relacionados à aplicação de reguladores vegetais, um manejo promissor para essa cultura. Informações sobre os efeitos destes produtos químicos na soja, poderiam fornecer elementos fundamentais para estudos posteriores da utilização agronômica dos reguladores vegetais.

STANKOVIC \& RAJKOVIC (1982) registraram ter sido a descoberta do Agrostemin feita por Danica Gajic, através de investigações sistemáticas sobre as interrelações alelopáticas, entre plantas de trigo e Agrostemina githago (L.).
Segundo GRINDBERG \& AZIMA (1986), plantas de tabaco pulverizadas com Agrostemin à $100 \mathrm{~g} / \mathrm{ha}$, num estágio de desenvolvimento onde apresentavam de 4 a 5 folhas, mostraram um aumento no peso total da planta, na área foliar e no número de folhas em relação à testemunha.

CASILLAS et al. (1986), tratando sementes de rabanete (Raphanus sativus L. cv. Red Prince) com Agrostemin, obscrvaram que houve aumento significativo na altura do vegetal.

CASTRO \& VELLO (1981) relataram que após 21 dias da aplicação de Agrostemin em soja, cultivar Davis, na concentração de $100 \mathrm{~g} / 1 / \mathrm{ha}$, a altura das plantas foi maior em relação às plantas

\footnotetext{
${ }^{1}$ Parte da dissertação de Mestrado, apresentada pelo $1^{\circ}$ Autor, ao Instituto de Biociências da Universidade Estadual Paulista - Campus de Botucatu em 1991 e financiada pela FAPESP.
} 
não tratadas. Quanto ao número de folhas, foi praticamente o mesmo das plantas testemunhas.

Por sua vez, CASTRO et al. (1987) estudaram em milho (Zea mays L. cv. C-515), os efeitos de Agrostemin. Verificaram que 0 estimulante vegetal, aplicado aproximadamente 45 dias pós-semeadura, não a fetou a altura das plantas.

$O$ presente trabalho teve por finalidade avaliar o efeito do Agrostemin sobre a altura e o número de folhas de plantas de soja (Glycine max (L.) MERRILL cV. IAC-8).

\section{MATERIAL E MÉTODOS}

O experimento foi conduzido em condições de casa de vegetação, no Departamento de Botânica do Instituto de Biociências/UNESP Campus de Botucatu.

Para a execução do trabalho, utilizou-se o produto comercial Agrostemin, tendo como principal composição química os seguintes aminoácidos: alantoina, triptofano, ácidos fólicos, ácidos alantóicos, ácidos glutâmico e adenina. Com essa substância, realizaram-se seis tratamentos, com três repctições, correspondentes às seguintes dosagens, épocas e formas de aplicação:

T1: Testemunha;

T2: 0,125g Agrostemin/100g sementes

T3: $0,125 \mathrm{~g}$ Agrostemin $/ 100 \mathrm{~g}$ sementes e pulverização foliar à $333 \mathrm{ppm}$;

T4: $0,125 \mathrm{~g}$ Agrostemin $/ 100 \mathrm{~g}$ sementes e pulverização foliar à $500 \mathrm{ppm}$;

T5: pulverização foliar à $333 \mathrm{ppm} \mathrm{e}$

T6: pulverização foliar à $500 \mathrm{ppm}$.

De acordo com as instruções encontradas na embalagem do produto comercial, sua aplicação foi feita diretamente na semente, a seco, previamente à semeadura. As pulverizações foliares foram feitas na pré-floração, ou seja, 43 dias após o plantio. Foram efetuadas três coletas de plantas (época I, II e III), a intervalo de 14 dias entre si.

A altura da planta é representada pela distância do colo até o último nó foliar, expressa em centímetros.

O delineamento experimental utilizado foi inteiramente casualizado. Realizou-se a análise de variância, com desdobramento em efeitos de regressão dentro de cada coleta, para os tratamentos T1, T5 e T6 e para os tratamentos T2, T3 e T4.

O nível de probabilidade adotado foi de $\alpha=0,05$; nas tabelas, os valores significativos a esse nível estão indicados com asterisco.

\section{RESULTADOS E DISCUSSÃO}

A observação das TABELAS 1 e $1 \mathrm{~A}$, mostra a ausência de significância para $o$ efeito de regressão. Entretanto, pela Figura 1, nota-se que há um aumento na altura das plantas, à medida que a concentração se eleva, sendo este mais acentuado na coleta III. Resultados similares foram demostrados por CASTRO \& VELLO (1981), ao estudar a ação deste estimulante vegetal aplicado via foliar em soja. O mesmo foi observado quando CASILLAS et al. (1986) empregaram Agrostemim a seco nas sementes de rabanete.

Os resultados obtidos para os tratamentos $\mathrm{T} 2$, T3 e $\mathrm{T} 4$, referentes a altura das plantas, são expostos nas TABELAS 2 e $2 A$, onde observa-se efeito significativo para regressão linear na coleta II, revelando uma redução drástica nessa medida, quando Agrostemin foi aplicado, tanto via semente como via foliar (T4). Talvez este decréscimo possa ser atribuído a uma concentração excessiva, a qual prejudicou o crescimento em altura das plantas.

São apresentados nas TABELAS 3 e $3 \mathrm{~A}$ e Figura 3 , os resultados obtidos para número de folhas, referentes aos tratamentos T1, T5 e T6, onde se observam efeitos significativos para regressão linear nas coletas I e II. $\mathrm{Na}$ coleta II, nota-se aumento considerável à medida que a concentração foi de 0 a $500 \mathrm{ppm}$. Estes resultados estão de acordo com GRINDBERG \& AZIMA (1986), porém discordantes daqueles de CASTRO \& VELLO (1981) e LACA-BUENDIA et al. (1984).

Através das TABELAS 4 e $4 \mathrm{~A}$, observase a não existência de significância para efeito de regressão, considerando-se os tratamentos $\mathrm{T} 2, \mathrm{~T} 3$ c T4.

Analisando-se a Figura 4, nota-se na coleta II, que as plantas que receberam 0 tratamento T3 mostraram valores superiores em relação aos demais tratamentos. Diante disso, pode ser sugerido que o Agrostemin, aplicado via semente e via foliar (333 ppm), foi suficiente para promover maior desenvolvimento das folhas. 
TABELA 1 - Análise de variância, com desdobramento em efeitos de regressão, para altura das plantas, considerando-se os tratamentos T1, T5 e T6 e as coletas I, II e III.

\begin{tabular}{lccc}
\hline \multicolumn{1}{c}{ Causa de Variação } & GL & QM & F \\
\hline Tratamentos & 2 & 202,04 & 1,90 \\
Coleta I & & & \\
R. Linear & 1 & 86,26 & 0,81 \\
R. Quadrática & 1 & 1,53 & 0,01 \\
Coleta II & & & \\
R. Linear & 1 & 26,04 & 0,24 \\
R. Quadrática & 1 & 3,12 & 0,03 \\
Coleta III & & & 3,54 \\
R. Linear & 1 & 376,04 & 0,59 \\
R. Quadrática & 1 & 62,35 & - \\
Resíduo & 18 & 106,30 & - \\
\hline T O T A L & 26 & 106,30 & \\
\hline \hline
\end{tabular}

TABELA 1A - Médias de tratamentos.

\begin{tabular}{cccc}
\hline \hline \multirow{2}{*}{ Tratamentos } & \multicolumn{3}{c}{ Coletas } \\
\cline { 2 - 4 } & I & II & III \\
\hline T1 & 85,33 & 90,83 & 89,00 \\
T5 & 90,00 & 91,67 & 01,33 \\
T6 & 92,92 & 95,00 & 104,83 \\
\hline \hline
\end{tabular}
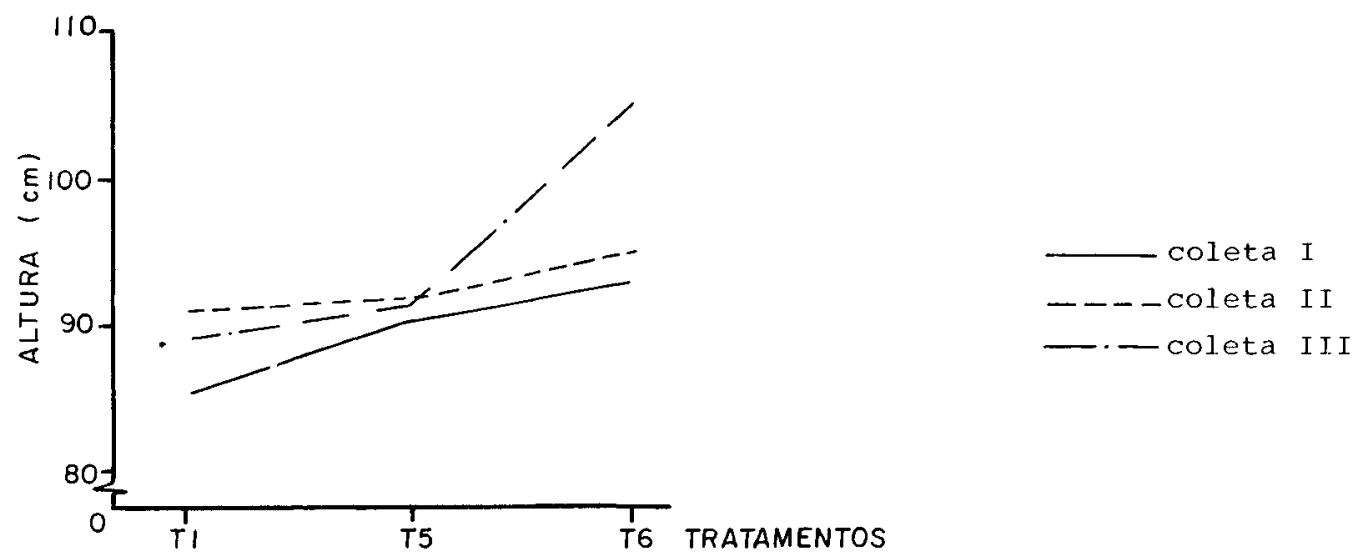

Figura 1 - Valores médios para altura das plantas, considerando-se as plantas que receberam Agrostemin via foliar.

Sci. agric., Piracicaba. 50(1):6-12, fev./maio, 1993 
TABELA 2 - Análise de variância, com desdobramento em efeitos de regressão, para altura das plantas, considerando-se os tratamentos T2, T3 e T4 e as coleta I, II e III.

\begin{tabular}{lccc}
\hline \multicolumn{1}{c}{ Causa de Variação } & GL & QM & F \\
\hline Tratamentos & 2 & 219,63 & 2,75 \\
Coleta I & & & \\
R. Linear & 1 & 15,04 & 0,19 \\
R. Quadrática & 1 & 8,68 & 0,11 \\
Coleta II & & & \\
R. Linear & 1 & 407,06 & $5,10^{*}$ \\
R. Quadrática & 1 & 152,66 & 1,91 \\
Coleta III & & & 1,31 \\
R. Linear & 1 & 104,17 & 0,08 \\
R. Quadrática & 1 & 6,72 & - \\
Resíduo & 18 & 79,75 & - \\
\hline T O T A L & 26 & & \\
\hline \hline
\end{tabular}

C.V. $=9,17 \% \quad *=$ Significativo a $5 \%$

TABELA 2A. Médias de tratamentos.

\begin{tabular}{cccc}
\hline \hline \multirow{2}{*}{ Tratamentos } & \multicolumn{3}{c}{ Coletas } \\
\cline { 2 - 4 } & I & II & III \\
\hline T2 & 96,33 & 105,50 & 101,33 \\
T3 & 92,67 & 106,00 & 99,00 \\
T4 & 93,17 & 98,03 & 93,00 \\
\hline
\end{tabular}

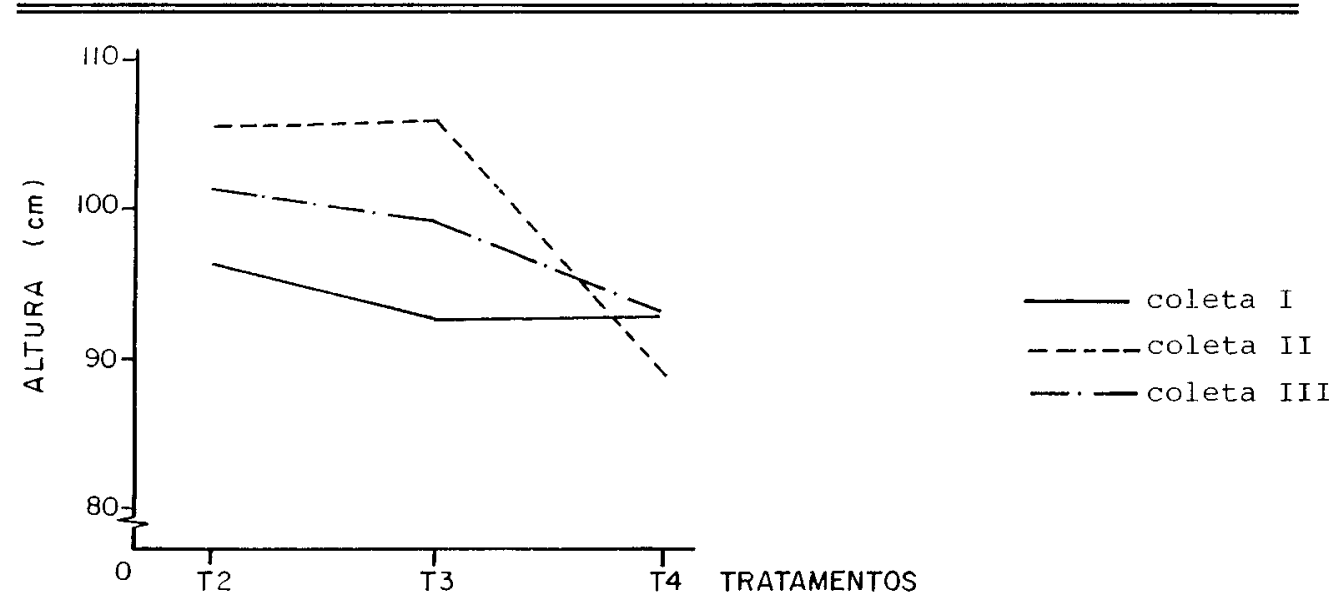

Figura 2 - Valores médios para altura das plantas, considerando-se as plantas que receberam Agrostemin, tanto por via foliar, como por semente. 
TABELA 3 - Análise de variância, com desdobramento em efeitos de regressão, para número de folhas, considerando-se os tratamentos T1, T5 e T6 e as coletas I, II e III.

\begin{tabular}{lccc}
\hline \multicolumn{1}{c}{ Causa de Variação } & GL & QM & F \\
\hline Tratamentos & 2 & 66,17 & $14,98^{*}$ \\
Coleta I & & & \\
R. Linear & 1 & 66,05 & $5,90^{*}$ \\
R. Quadrática & 1 & 8,68 & 1,97 \\
Coleta II & & & \\
R. Linear & 1 & 117,04 & 26,50 \\
R. Quadrática & 1 & 1,68 & 0,38 \\
Coleta III & & & 3,41 \\
R. Linear & 1 & 15,04 & 0,91 \\
R. Quadrática & 1 & 4,01 & - \\
Resíduo & 18 & 4,42 & - \\
\hline T O T A L & 26 & & \\
\hline \hline
\end{tabular}

C.V. $=7,53 \% *=$ Significativo a $5 \%$

TABELA 3A. Médias de tratamentos.

\begin{tabular}{cccc}
\hline \multirow{2}{*}{ Tratamentos } & \multicolumn{3}{c}{ Coletas } \\
\cline { 2 - 4 } & I & II & III \\
\hline T1 & 23,17 & 25,83 & 26,17 \\
T5 & 27,33 & 31,17 & 26,33 \\
T6 & 27,33 & 34,67 & 29,33 \\
\hline \hline
\end{tabular}
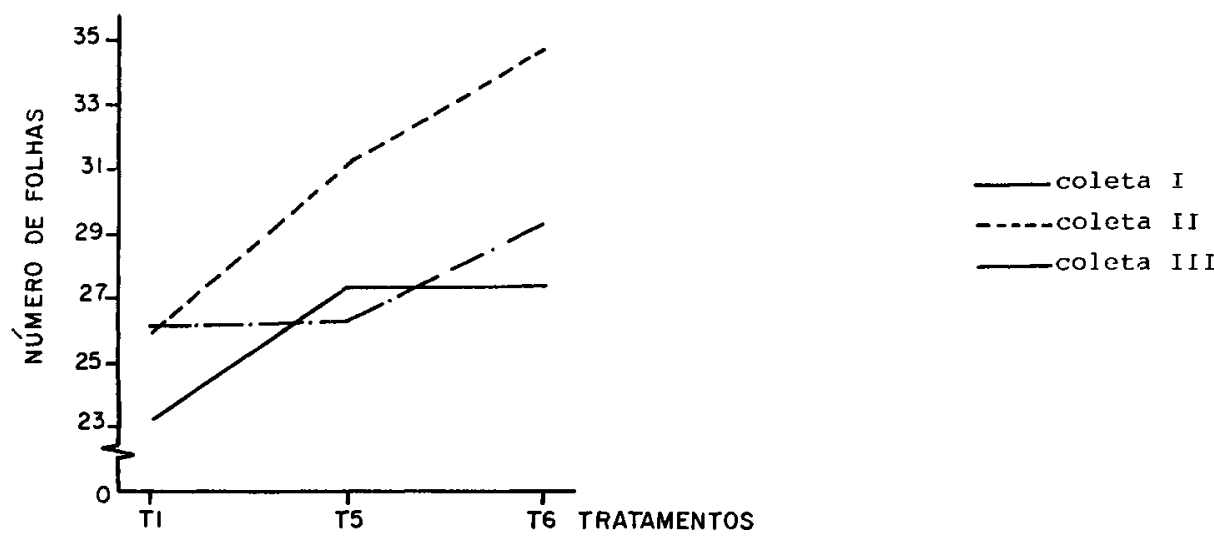

Figura 3 - Valores médios para número de folhas, considerando-se as plantas que receberam Agrostemin via foliar. 
TABELA 4 - Análise de variância, com desdobramento em efeitos de regressão, para número de folhas, considerando-se os tratamentos $\mathrm{T} 2, \mathrm{~T} 3 \mathrm{e} \mathrm{T} 4 \mathrm{e}$ as coletas I, II e III.

\begin{tabular}{lccc}
\hline \multicolumn{1}{c}{ Causa de Variacão } & GL & QM & F \\
\hline Tratamentos & 2 & 2,90 & 0,51 \\
Coleta I & & & \\
R. Linear & 1 & 1,50 & 0,26 \\
R. Quadrática & 1 & 0,89 & 0,16 \\
Coleta II & & & \\
R. Linear & 1 & 8,17 & 1,43 \\
R. Quadrática & 1 & 16,05 & 2,82 \\
Coleta III & & & \\
R. Linear & 1 & 2,67 & 0,47 \\
R. Quadrática & 1 & 0,22 & 0,04 \\
Resíduo & 18 & 5,69 & - \\
\hline T O T A L & 26 & & - \\
\hline \hline
\end{tabular}

C.V. $=8,41 \%$

TABELA 4A. Médias de tratamentos.

\begin{tabular}{cccc}
\hline \hline \multirow{2}{*}{ Tratamentos } & \multicolumn{3}{c}{ Coletas } \\
\cline { 2 - 4 } & I & II & III \\
\hline T2 & 26,17 & 31,33 & 25,67 \\
T3 & 25,00 & 35,33 & 26,00 \\
T4 & 25,17 & 33,67 & 27,00 \\
\hline \hline
\end{tabular}
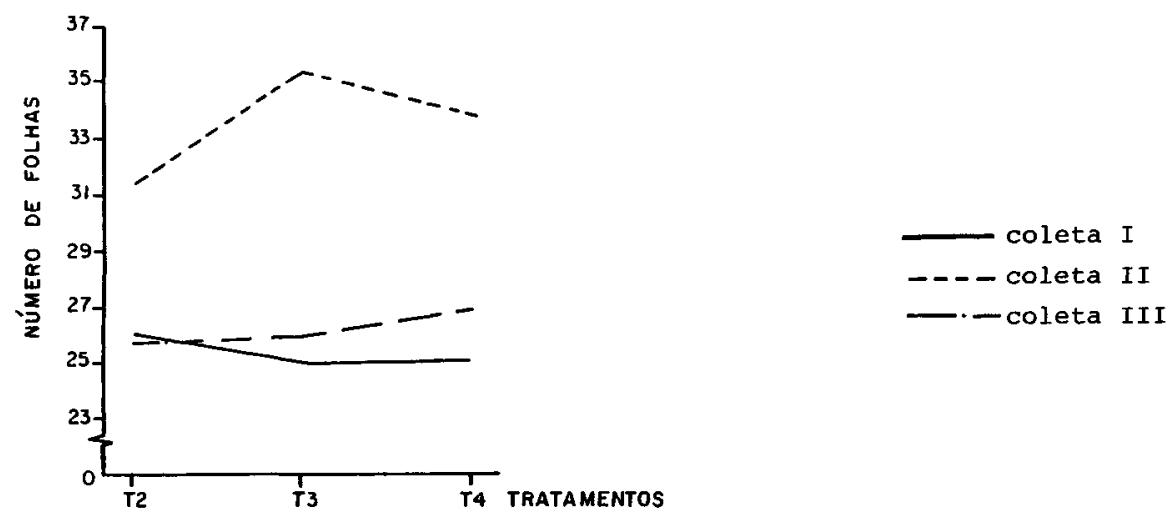

Figura 4 - Valores médios para número de folhas, considerando-se as plantas que receberam Agrostemin, tanto por via foliar como por semente. 


\section{CONCLUSÕES}

Concluiu-se que:

- as plantas que receberam Agrostemin via semente (T2) ou via foliar a $500 \mathrm{ppm}$ (T6), apresentaram maiores valores para altura e número de folhas.

- Agrostemin quando aplicado via semente mais via foliar a $500 \mathrm{ppm}(\mathrm{T} 4)$, promoveu decréscimo, tanto na altura como no número de folhas.

\section{REFERÊNCIAS BIBLIOGRÁFICAS}

CASILLAS, J.C.; LONDONO, J.; GUERREIRO, H.; BUITRAGO, L.A. Análisis Cuantitativo de la aplicacion de cuatro bioestimulantes em el cultivo rabano (Raphanus sativus L.). Acta Agronomica, Palmira, v.36, p.185-195, 1986.

CASTRO, P.R.C. Efeitos de fitorreguladores na produtividade da soja (Glycine max cv. Davis) em competição. Anais da Escola Superior de Agricultura "Luiz de Queiroz", Piracicaba, v.38, n.1, p.289-298, 1981.

CASTRO, P.R.C.; VELLO, N.A. Açāo de fitorreguladores no desenvolvimento da soja cultivar Davis. Anais da Escola Superior de Agricultura "Luiz de Queiroz", Piracicaba, v.38, n.1, p.269-279, 1981.
CASTRO, P. R. C.; CONFORTO, E.; NICOLINI, E.M.; GABRIEL, J.L.C.; ISMAEL, J.J. Efeitos de reguladores e estimulantes vegetais no desenvolvimento do milho (Zea mays L. cv. C-525). Anais da Escola Superior de Agricultura "Luiz de Queiroz", Piracicaba, v.44, n.2, p.1079-1105, 1987.

GRINDBERG, I.P.; AZIMA, A.V. Stimulation of growth to tobacco vegetative organs by Agrostemin. Tabak, Kishinev, v.1, p.12-14, 1986. 620, 1986. (Resumo).

LACA-BUENDIA, J.P.; RAFAEL, J.O.; TANAKA, R.T. Efeito de bioestimulante na cultura da soja. In: SEMINÁRIO NACIONAL DE PESQUISA DE SOJA, 3., 1984, Londrina. Londrina: EMBRAPA/Centro Nacional de Pesquisa de Soja, 1984, p.27-33.

STANKOVIC, D.; RAJKOVIC, N. Agrostemin: a new factor of horticultural production. In: INTERNATIONAL HORTICULTURAL CONGRESS, 21., 1982, Hamburg. Proceedings... Hamburg, International Society for Horticultural Science, 1982. p.2125.

Trabalho entregue para publicação em 20.01 .92 Trabalho aprovado para publicação em 25.03.92 